\title{
Téoros
}

Revue de recherche en tourisme

\section{Le tourisme international à l'horizon 2000 : quantité ou qualité ?}

\section{Pierre Gouirand}

Volume 12, numéro 2, juillet 1993

La prévision-prospective du tourisme

URI : https://id.erudit.org/iderudit/1078022ar

DOI : https://doi.org/10.7202/1078022ar

Aller au sommaire du numéro

Éditeur(s)

Université du Québec à Montréal

ISSN

0712-8657 (imprimé)

1923-2705 (numérique)

Découvrir la revue

Citer cet article

Gouirand, P. (1993). Le tourisme international à l'horizon 2000 : quantité ou qualité ? Téoros, 12(2), 22-26. https://doi.org/10.7202/1078022ar d'utilisation que vous pouvez consulter en ligne.

https://apropos.erudit.org/fr/usagers/politique-dutilisation/ 


\title{
Le tourisme international à I'horizon 2000 : quantité ou qualité?
}

\author{
Pierre Gouirand ${ }^{*}$
}

En $1990,1^{\prime} \mathrm{OMT}$ a recensé 411 millions d'arrivéesinternationales de touristes. Quel'on prenne l'hypothèse basse ou l'hypothèse haute, il y en aura au début du XX广* siècle, entre 600 et 800 millions.

Le monde économique et politique se réjouit de cette perspective.

En période de récession, l'industrie du tourisme reste crétrice d'emplois et de nombreux pays en voie de développement fondent de grands espoirs sur l'augmentation du nombre de touristes. Ils se disent qu'ils récolteront certainement une part importante de ces nouveaux voyageurs car les destinations traditionnelles étant saturées, c'est vers eux naturellement que l'on dirigera le surplus de touristes.

Les pays développés se réjouissent aussi. On a, en effet, beaucoup construit d'hôtels et de structures d'hébergement dans les capitales et dans les départements d'outremer, souvent pour des raisons qui faisaient abstraction de la rentabilité directe. En France, par exemple, pour profiter des mesures fiscales tavorables et dites de «défiscalisation», ailleurs pour profiter de permis de construire, pour conforter la politique d'expansion des transporteurs ou encore simplement pour créer des retombées économiques indirectes.

Les grands transporteurs, entraînés par la concurrence, ont «dérégulés les tarifs, c'està-dire, qu'ils ont fait baisser les prix, touten augmentant les fréquences et la taille des avions.

Il résulte de toutcela qu'ily a de plusen plus de lits touristiques et de places d'avions à vendre. On comprend donc que le monde du tourisme se réjouisse de savoir que le nombre d'arrivéesinternationalesdoublera dans les années à venir.

Or, la réalité est bien différente. Tout le monde ne va pas profiter de l'expansion. prévue. Ce sont, au contraire, les destinations traditionnelles qui, peu à peu, deviennent de wéritables sanctuaires touristiques, qui vont bénéficier principalement (ou pâtir)

Monsieur Pierre Gouirand est président de l'lins. titut de Prospective du Tourisme. Nice, France. de cette formidable expansion. En effet, il est très difficile de répartir spatialement et chronologiquement les flux touristiques, et au contraire, il semble que l'on aille vers une hyper-concentration du tourisme.

On est entré dans une sorte de cycle infernal, où pour utiliser les équipements existants, on a incité le public à voyager davantage, ce qui a entraîné la nécessité de construire toujours plus d'équipements.

Une énorme machine économique, composée de publicitaires, d'agents de voyages de toutessortes: toursoperators, détaillants, autocaristes, réceptifs d'hôteliers, de restaurateurs, de transporteurs, d'institutionnels: office du tourisme, services gouvernementaux, de tous ceux qui voient dans le tourisme un moyen de faire de l'argent, s'est construite et s'est mise en mouvement au nom du développement. Elle s'auto-alimente et son fonctionnement est devenu incontrôlable.

Le monde des affaires et de la culture s'est aussi joint au mouvement général et ne cesse d'appeler ses gens ầ participer à des congrès, colloques ou à des rencontres de toutes sortes qui mettent encore plus de monde sur les routes.

Pour encore augmenter le nombre de touristes potentiels, on a fait baisser les prix de façon à ce que des couches sociales, de moins en moins favorisées, puissent se déplacer et acheter des «produits touristiques\%. Il est donc vraisemblable que les prévisions de l'OMT se réaliseront, mais les espoirs des nouvelles destinations, en revanche, risquent bien d'ètre déçus.

Certes, une partie de ce nouveau marché du tourismeira vraisemblablementversces nouvelles destinations et se répartira dans le monde, mais hélas, la plus grande partie de ce marché ira, au contraire, vers les grands centres touristiques traditionnels.

En effet:

- tous les *nouveaux touristese, avant de penser à aller se reposer dans un coin tranquille, veulent visiter ce que nous avons appelé les ssanctuaires touristiquesw: Paris, Rome, Venise, etc... 200 ou 300 points dans le monde qui ont la réputation d'être très beaux, très intéressants ou d'être des lieux de culture ou de loisir (le tourisme des groupes japonais en Europe est en ce sensexemplaire: avant devoir le Larzac ou les Fjords norvégiens, ils visitent les capitales);

- ces grands centres agissent comme des xaimants\%, même pour les touristes chevronnés. On peut aller 10 fois à Paris, Rome ou Venise, sans en épuiser les ressources et il semble au contraire que plus on $y$ va, plus on a envie d'y retourner;

- le développement des transports se fait surtout en direction des grandes concentrations urbaines, qui sont pour la plupart de grands centres de tourisme: New York, Londres, Paris, etc.

Les aćroports deviennent des ahub», des plaques tournantes géantes qui permettent de adistribuero les passagers vers d'autres *hub* et où les compagnies aériennes trouvent des facilités de traitement et d'accueil et beaucoup plus de clients que dans les petits aéroports. Elles ont donc tendanceà concentrer leurs vols vers ces «hubs.

Il en est de même pour les transports ferroviaires et les transports routiers. Le développement des premiers se fait autour des trains à grande vitesse, qui desservent en priorité les grandes villes, les seconds autour d'autoroutes, la plupart du temps à péage, qui pour être rentables doivent relier des centres très actifs où il y a beaucoup de trafic et au contraire éviter les endroits wcalmess.

Ainsi, on constate que les grands centres touristiques sont beaucoup plus faciles d'accès que les destinations de petites capacités et que grâce au nombre de passagers voyageant sur ces axes, les prix y sont bien plus avantageux que sur les lignes moins fréquentées. Il est, par exemple, guère plus cher d'aller de Parisà New YorkquedePau à Strasbourg.

Malgré une tendance dans l'hôtellerie de grand luxe à diminuer le nombre de chambres des unités hôtelières, l'hôtellerie de grand tourisme continue à construire des hôtels de très grande capacité, ce qui revient moins cher pour le même nombre de chambres que des hôtels de petite capacité. 
Ces hôtels sont concentrés géographiquement dans des quartiers prevus å cet effet, par exemple autour de Disneyland à Marne la Vallée, Hôtel Circle à San Diego ou le bord du lac à Bordeaux.

La satisfaction des nouvelles exigences du publić, dues à l'enrichissement quasi général des populations des pays émetteurs et à leur goût pour le confort qui en résulte (toutes les chambres doivent avoir des salles de bains, l'air conditionné, les rues doivent être bien éclairées, les plages équipées, etc.) et l'obligation de construire tous les équipements sophistiqués au moindre coût, impliquent forcément la création de *mégapoles touristiques*.

Cen'estquedans des centrès suffisamment importants que l'on peut rentabiliser, non seulement les moyens d'accès (aéroports, ports, gare TGV, autoroutes, etc.), mais aussi les équipements ludiques (parc d'attractions, remontées mécaniques, golf, spectacles), les équipements commerciaux (boutiques de luxe, excursions, night club, etc.) et les structures d'accueil (palais des congrès, hébergements divers, office du tourisme, bureaux de renseignements).

Il y a une course permanente entre les exigences des touristes et les destinations, qui sont contraintes d'investir toujours plus pour satisfaire leur clientèle.

Comme l'équilibre financier ne peut être atteint que si les équipements *tournent à fond», on est bien obligé de diriger les touristes vers ces *mégapoles», acceptant ainsi le phénomène de concentration touristique. Des bourgades, comme Ouarzazate dans le sud Marocain, qui était un village de quelques centaines d'âmes sur les circuits des Casbah entre Zagora, Timerhir et Erfoud, sont transformées en centre de tourisme et ont maintenant un aéroport qui peut accueillir des Boeings et plusieurs centaines de chambres d'hotel. C'est à ce prix que le public peut bénéficier des techniques modernes de confort (réfrigérateur, électricité, route goudronnée, etc.).

Par ailleurs, il y a aussi concentration du tourisme dans le temps. Les pays émetteurs de touristes sont, pour le moment, des pays riches de l'hémisphère nord. Des raisons climatiques, et aussi comportementales, font que la grande majoritédes individus prend des vacances pendant la même période. En France, tout au moins, toutes les tentatives d'étalement des vacances ont été vouées a l'échec et la plupart des congrèset des colloques se concentrent sur six mois de l'année, automne et printemps.

Enfin, il faut noter que seuls les États riches, stableset solides ont la possibilité de mettre en oeuvre une politique du tourisme réellement efficace. Seuls, ils ont les moyens financiers, d'équiper la totalité du territoire. Les Etats pauvres du tiersmonde, qui doivent compter sur des financements extérieurs (Banques mondiales, FMI, etc.) ne peuvent équiper que des points touristiques. Leur tourisme d'ailleurs ne repose en général que sur un seul type d'attraction: le soleil, la plage, la chasse ou des ruines. Ce sont des destinationsà mono-motivations touristiques, par opposition aux pays riches qui jouissent de nombreux attraits pour attirer les touristes: culture, gastronomie, équipements, monuments, histoire, etc. Même si ces pays pauvres ont un certain succès touristique à leur échelle, il n'est en aucune façon comparable à celui des grands pays riches, traditionnellement récepteur de tourisme (France, Angleterre, Italie, etc.).

Les pays riches ont, en outre, la possibilité de créer des réseaux de vente extrêmement efficaces (bureaux de tourisme à l'étranger, compagnies de transports, accord avec des représentants et de nombreux tours operators, etc.), de faire beaucoup de publicité (presses, cinéma, démarchage à l'étranger, accueil de décideurs, etc.).

Ils peuvent aussi mettre en place des politiques d'incitation à l'investissement (défiscalisation, primed'équipement, etc.). Ils ont par ailleurs pu créer une législation adaptée au tourisme (normes hốtelières, réglementation pour les agences de voyage, sécurité dans tous les domaines: bien, personnes, hygiène, transports, etc., changes, douanes, etc.).

Ainsi, malgré ce que l'on pourrait croire au premier abord et en dépit des coûts de main-d'oeuvre élevés, les pays développés sont très avantagés et reçoivent de plus en plus de touristes, alors que les pays pauvres, qui en auraient le plus besoin, malgré une main-d'oeuvre bon marché, une authenticité certaine et des prix attractifs, ont beaucoup de difficultés â décoller.

Lecycle estinfernal, en ce sens qu'il engendre une concentration touristique toujours plus grande. On fait de la publicité pour élargir le marché du tourisme avec l'espoir d'aiderdesdestinations défavoriséesetc'est vers les grands centres queva le public, car ils sont faciles d'acoès, mieux équipés, plus agréables, etc., et seuls quelques ascètes ou aventuriers sortent des sentiers battus.
On crée donc de plus en plus d'équipements dans ces centres où il faut toujours plus de monde pour les rentabiliser. L'énorme machine économique touristique continue de fonctionner dans une seule direction, celle de la facilité (des marchés porteurs comme on dit), sans se rendre compte qu'elle risque de courir à sa perte car quoi que l'on fasse, on s'aperçoit que le phénomène progresse.

Les effets pervers du tourisme ont été maintes fois dénoncées, mais il semble que l'on ne se soit pas rendu compte de l'inexorabilité de la concentration et que c'étaitcetteconcen tration qui était responsable du surnombre de touristes dans les mégapoles, sanctuaires touristiques, et que cela constituait un véritable danger pour le tourisme.

\section{Quantité - qualité}

Bien que le phénomène touristique soit né de l'industrialisation et de la commercialisation de la tendance naturelle de l'homme à voyager, à connaître et à jouir des paysages, il est difficile de concilier quantité et qualité dans le tourisme.

La qualité maximum de la prestation économique relève del'artisanat et ne peut pas être atteinte par l'industrialisation. La production de masse s'oppose à la perfection, sinon dansl'absolu, certainement dans la pratique. On n'a pas trouvé le moyen de produire un plat à 10000 exemplaires aussi parfaitement que pour 10 personnes. Le grand nombre implique l'uniformisation de la prestation (la decoration des chambres d'hôtel est neutre, le café est toujours mauvais car s'il était bon pour l'italien, il serait imbuvable pour l'Américain, quand tout le monde le trouve mauvais, tout le monde se plaint mais tout le mondele boit; et les «self service», le long des autoroutes, n'ont pas remplacé les bistrots pour «routiers» des nationales).

Le grand nombre dégrade la sensation et l'environnement. On ne peut goûter avec autant d'intérêt et de plénitude la vue d'un tableau ou d'un monument si on est au milieu d'une foule qui vous pousse et vous bouscule. Il faut, pourapprécier uneoeuvre d'art, un certain recueillement, impossible à trouver dans la multitude. Un paysage désert a souvent une majesté et une beauté qui disparaissent dès que les paysage est envahi par l'homme. La moindre plage, la moindre rue pittoresque ou n'importe quelle forêt, perdent tout intérêt si la foule sy presse. 
L'influence de la quantité est encore plus sensible dans les grandes villes, car s'il a été possible de développer les structures d'hébergement, d'augmenter le nombre des places dans les restaurants et de construire des parkings, il n'a pas été possible, en revanche, d'agrandir les points d'interêts touristiques: le Louvre ou la rue du Faubourg Saint Honoré à Paris, le Colisée ou la Via Condottià Rome, le Grand Canal ou le Pont des Soupirs à Venise. Ainsi, on arriveen *saison» à un engorgement deces merveilles du monde qui les banalise et les transforme en objet de consommation de masse.

La quantité toutefois ne dégrade pas toujours l'objet touristique. Un tableau ne souffre pas d'avoir 1000 personnes autour. Une foule ne détruit pas toujours matériellement un paysage. Mais le nombre dégrade la sensation. La perception d'un tableau comme celle d'un paysage est modifiée par le nombre de personnes qui les regarde et qui sont autour de celui qui les perçoit. La présence des autres, sans abîmer l'objet, abîme la vue de l'objet.

Le fait que l'on se bouscule sur la Grande Muraille de Chine ne permet plus d'en apprécier la dimension, de réaliser l'ampleur de l'ouvrage.

Le surnombre de touristes:

1) amène des effets pervers de toutes sortes:

- le maire de Venise a dû fermer l'été dernier sa ville aux excursionnistes de la journée;

- le gouvernement du Cambodge se demande si le site d'Angkor Vat sera ouvert aux touristes, compte tenu des risques que fera courir aux monuments la fréquentation d'un grand nombre de personnes;

- un grand journal régional a titré le 9 août 1992: *un nouvel embarras pour les rues de Paris: le car de tourismen, et cite le maire qui déclare: sla situation devient intolérable». Le même journal, deux jours plus tard, publie une photo montrant l'esplanade du Champ de Mars couverte de sacs de couchage et a indiqué que tous les jours, à 5 h 30 du matin, des policiers devaient venir déloger les dormeurs pour rendre la place à sa destination (Nice Matin, 9 et 11 août 1992).

2) ou crée des situations intolérables:

- l'accèsà tous les monuments doit être réglementé et les files d'attentes s'al= longent. Il faut parfois une heure pour entrer au Louvre et il fallait plusieurs heures pour pénétrer dans certains pavillons lors de l'Exposition Universelle de Séville de 1992;

- en mai 1992, quelques 20000 *hippies» ont envahi pour le weekend un petit village de l'ouest de l'Angleterre, saccageant tout sur leur passage (seule la presse anglaise en a fait mention, etencore très discrètement);

- lorsdesa faris au Kenya, les lions(peutêtre plus intelligents que les hommes) ont compris qu'en suivant les nombreuses voitures qui amenaient les touristes, ils trouveraient des gazelles dont ils pourront se régaler.

Ainsi, la quantité attente à la qualité, ce qui entraîne à court terme des effets de régulation dontles conséquences sont souvent dramatiques.

On connaît la théorie du cycle de vie des stations touristiques:

lère phase: les découvreurs, qui sont souvent des artistes ou des intellectuels, s'installent dans un endroit qu'ils aiment.

2ème phase: les riches investisseurs yviennent, attirés par la réputation qu'ont donné au lieu les découvreurs. Ils y créent une vie mondaine et brillante dont profitent quelque temps les déoouvreurs.

3 ème phase: le grand public entend alors parler de la *station\%. Les touristes arrivent. Les découvreurs partent. Les investisseurs restent le temps d'amortir leurs investissements. Quand cela devient insupportable, ils s'en vont. Les touristes baissent de qualité et la station meurt. Parfois, elle est redécouverte. Quelquefois, elle ne se relève jamais.

Dans une station, une grande affluence a souvent pour effet de faire monter les prix, ce qui est rédhibitoire pour le tourisme de masse, basé sur des conditions tarifaires attrayantes. Ce fut le cas de l'Espagne, il y a deux ans et del'Italie cet étéqui annonçaít une diminution du nombre de touristes de $20 \%$ en général et de $30 \%$ à Rome en particulier (Nice Matin du 21 août 1992).

Ces crises d'autorégulation sont très dangereuses économiquement car elles entraînent de nombreuses perturbations, notamment la diminution des marges. On sait que la construction des structures d'hébergement, pour le tourisme de masse, est faite grâce à l'emprunt, ce qui rend nécessaire de forts taux d'occupation et entraîne souvent une réduction des services, afin de dégager suffisamment de béné- fice pour pouvoir payer les loyers de l'argent. Dès que le public, lassé par la baisse de qualité ou attiré par une autre destination, pour des questions économiques ou des questions de mode, déserte quelque peu ces destinations, il y a crise avec les conséquences économiques et socio-économiques que cela entraîne.

Ainsi, nous sommes face à un des grands paradoxes del'époque moderne. L'homme domestiqué par la société de consommation, enrégimenté par la division du travail dans de grandes organisations, aspire pour sesvacances et ses loisirs à se retrouver, non pas complètement seul, car Pascal l'a bien montré, il ne le supporterait pas, mais dans un environnement à sa mesure où il prend conscience de lui, où il peut jouir de la compagnie des autres plutôt que d'y être soumis.

Or, le succès du tourisme fait qu'on ne lui offre plus qu'une transposition, en terme de tourisme, de la société de consommation et de ses grands nombres. Cette situation dedéséquilibre paradoxal est tout compte fait angoissante. Nous aurons, d'une part, une croissance du marché touristique, de 150 à 350 millions d'arrivées internationales, plus le trafic national très important dans les pays développés. Cette augmentation du marché devrait permettre de faire face à la croissance de loffre et d'arriver à un bon équilibre.

Mais d'autre part, ces données sont bouleversées par la tendance des touristes à se concentrer en certains points du globe. Nous allons sans doute vers une asphyxie des grandes destinations touristiques traditionnelles, alors que d'autres resteront vides ou presque.

Ce qui est paradoxal, c'est que la progression du nombre de touristes au lieu de contribuer à rétablir un certain équilibre va, au contraire, accentuer le déséquilibre existant etainsi multiplier les effetsnégatifs du nombre sur la qualité des sites et des prestations.

Pour employer le langage du marketing, on peut dire que le succés du produit touristaque détruit le produit lui-même, puisque ce que l'on vend est fait, en partie, de l'environnement naturel, de l'environnement humain et de l'équilibre de ces deux composants. Quand cet équilibre est rompu, notamment par l'augmentation du nombre des humains, il y a dégradation du produit. Par exemple, on peut parler d'hôtel de luxe autant que les clients ont un certain comportement et sont en nombre compatible avec les lieux. Si l'hôtel est 
envahi par une foule sans éducation et que l'on met 10 personnes par chambre, on ne peutplus parler d'hôtel de luxe, même si les lieux n'ont pas changé.

Pour rester ce qu'il est, et si possible pour s'améliorer, le phénomène touristique va devoir se réguler. Mais cette régulation ne peut pas être subie, c'est-à-dire produite par les crises, elle doit être voulue et contrôlée.

Ce sera le défi du XXIt siècle que de pouvoir gérer en matière de tourisme la quantité et la qualité, et de savoir maintenir l'équilibre entre les deux car, sans une certaine quantité de touristes, pas de qualité possible, parce que pas de marché, par d'argent, aucun équipement, aucune animation. Mais dès que le seuil de stolérances est atteint, on bascule dans la dégradation.

Cette théorie implique des choix politiques dramatiques, qui souvent se résument en un seul choix: le court terme ou le long terme.

Si on a choisi le court terme, tout est très facile, on libère tout, on laisse construire n'importe quoi, on fait de la publicité, on tâche de gagner beaucoup d'argent le plus rapidement possible et quand la source est tarie, on va ailleurs. Ce choix est possible pour les stations créées de toutes pièces et éphémères, il devient impossible pour les *sanctuaires touristiques", les capitales, les pays, les paysages.

L'homme est comptable devant l'humanité de ce qu'il fait, de ce qui lui est confié. Le proprietaire d'une terre n'en est, en définitive, que dépositaire et si le droit positif peut lui permettre de la saccager, le droit naturel le lui interdit. Il doit la restituer à ses descendants, au moins dans le même état sinon en meilleur état que celui dans lequel elle lui a êté transmise.

Les dirigeants politiques doivent veiller à ce que le patrimoine touristique survive et ils doivent être en ce sens soutenus par les professionnels et aussi par le public.

L'homme de tourisme est responsable du produit vendu. S'il dit à quelqu'un: «Venez visiter le Louvres, encore faut-il que ce client puissey pénétrer normalement, qu'il puisse admirer ce qui est exposé pendant le temps souhaité et qu'il puisse y revenir aussi souvent qu'il le veut. S'il en est empêché, cela correspond à une tromperie et frise l'escroquerie. C'est pourtant ce à quoi amène un trop grand nombre de touristes.
Mais le problème est de taille. Comment faire pour maintenir ou canaliser la marée montante des futurs touristes? Comment dire Paris ne doit recevoir que $\$ 0 \%$ milliers de touristes en même temps? Comment dire seulement $* x \approx$ centaines de personnes pourront voir le Colisée chaque jour? Comment dire que pour qu'un voyage soit agréable, il faut que l'aéroport où on atterrit ait telle taille, ce qui veut dire qu'il ne recevra que sxo centaines de milliers de personnes au lieu de $* x \neq$ millions.

Comment dire, mais aussi comment faire? Sur quels critères fonder la sélection? Comment organiser ce Malthusianisme touristique? En admettant que l'on puisse le dire et peut-être le faire, a-t-on le droit de le dire et de le faire? La liberté de voyager est une des libertés fondamentales de l'homme et il est inconcevable de l'en priver donc, a-t-on le droit de décréter que seul un petit nombre de personnes pourra voir tel paysage, visiter tel endroitou prendre le soleil sur telle plage?

L'entreprise privée a en partie résolu ce dilemme. Quand un restaurant est complet, on ne prend pas un client de plus et le restaurateur, malgré son désir d'augmenter ses profits, ne rajoute pas indéfiniment des sièges car cela gêneraitles autres clients qui partiraient ou ne reviendraient plus. Un équilibre est ainsi créé entre la qualité et la quantité, et pour reprendre ce même exemple, plus la qualité du restaurant est élevée, plus on a d’espace, donc moins il y a de personnes au mètre carré. Ledilemme est facile à résoudre car il y a l'autorité sans appel du chef d'entreprise.

Dans le cas des espaces ouverts au public, comme un musée, une ville, un paysage, l'approche est différente. Il y a d'abord un problème de mesure. Le restaurateur a un nombre déterminé de places. Mais combien de personnes peut normalement contenir la Place de la Concorde et surtout à partir de combien de personnes est-il *inconfortables de séjourner Place de la Concorde et aussi à partir de combien de personnes le paysage de la Place de la Concorde est-1 1 gâché? Pendant combien de temps est-il raisonnable de faire la queue pour entrer au Louvre?

Ensuite, probleme de liberté. Au nom de quoi, s'il n'y a pas dégradation matérielle, interdire à quelqu'un, qui accepte d'être dans la foule et qui estime qu'elle ne gẫche pas le paysage, de venir Place de la Concorde rejoindre les 250000 personnes qui y sont déjà et qui préferent la voir ainsi plutôt que de ne pas la voir du tout?
Enfin, probleme politico-sacial. Que dire aux laissés pour compte: ceux à qui on aurait interdit d'aller Place de la Concorde et qu'en faire?

Il faut aussi prendre en compte la dimension éducative du tourisme. La visite de lieux et de monuments fait partie de l'éducation. C'est mêrne ce qui est à l'origine du tourisme (le fameux tour que faisait aux $\mathrm{XVIII}^{e}$ siècle les jeunes aristocrates britanniques à l'issue de leurs études). Donc, comment remplacer culturellement l'expérience unique du contact direct et physique avec une ville, une population ou un objet. Traditionnellement, le voyageur $s$ 'est toujours enrichi de ce qu'il ramenait d'expérience et de connaissance. Supprimer une telle source de savoir pour un certain nombre est aussi une bien grave responsabilité.

Nous voyons donc la succession de problèmes qui pourraient surgir d'un Malthusianismetouristique: économiques, pratiques, moraux, politiques, sociaux, etc.

Pourtant, tout le monde en est bien conscient. La quantité tue la qualité et si nous voulons continuer à faire du tourisme, dans le sens où nous l'entendons, c'est-à̀-dire jouir des choses et des gens, del'environnement et des paysages, ou seulement utiliser les équipements qui sont mis à notre disposition, il nous faudra réguler les flux touristiques. Cette régulation deviendra indispensable car comme nous l'avons mentionné:

a) le nombre de touristes potentiel croit plus rapidement que la capacité d'accueil des grands centres récepteurs;

b) malgré les efforts apparents de décentralisation touristique (création de stations et de destinations), tout tend à diriger les flux vers les mêmes points d'intérêts.

De plus, comment faire accepter cette nécessaire récession aux professionnels du tourisme et aux investisseurs, alors qu'ils espèrent au contraire une expansion colossale? Comment faire admettre à certains qu'ils ne verront jamais Paris ou Rome, même s'ils en ont le temps ou l'argent?

C'est cette tâche qui attend les administrateurs du tourisme de la prochaine décennie.

Elle sera d'autant plus difficile que les progrès techniques continueront à rendre les voyages plus faciles et moins chers, que les médias continueront à donner au public l'envie de se déplacer et que les stratèges de 
la société de consommation le pousseront à consommer toujours plus (de tourisme).

La réponse sera forcément à la fois réglementaire et culturelle. Rien ne pourra se faire brutalement. On ne passera pas d'un seul coup, comme en temps de crise de l'expansionàladépression. Il faudra d'abord sans doute réguler la courbe ascendante, la stabiliser et peu à peu, au fur et à mesure que les pays pourront se reconvertir, faire décroitre le nombre de touristes en certains points qui, saturés allaient vers l'asphyxie. Peut-être d'ailleurs que ces mesures, appliquées aux grandes destinations, aideront les destinations secondaires à se remplir?

Si on a assisté à une très grande concentration des moyens de transportet des moyens d'hebergementen quelques pointsduglobe, c'est parce qu'il y a eu combinaison des motivations de voyages: affaire, culture, loisir, religion, etc. Les grands centres d'affaires (Paris, Londres, New York) sont aussi de belles etgrandes villes où on trouve les plus beaux musées, les meilleurs opéras etles plus grands restaurants. On nepourra guèreagir et cene serait pas souhaitablesur Pattraction qu'exercent ces centres.

En revanche, réglementairement, on peut agir. Agir sur le développement des transportset desstructures d'hébergement, être plus sélectif pour les autorisations de vol, les accès par train et par voiture, plus difficile pour les permis de construire d'hôtel, etc. Certaines grandes villes américaines, apres avoir fait une politique qui tendait à faciliter l'acces des automobiles, font maintenant machine arrière. On réduit les voies de circulation, on y interdit la construction de parkings, le stationnement sur la woie publique et les amendes sont très élevées. Parallèlement, on a amélioré les transports publics et augmenté le nombre de taxis. Les résultats sont, paraît-il assez encourageants et le nombre de voitures tend à diminuer.

Nous serons sans doute contraints d'adopter ce type de mesures réglementaires pour le tourisme. Les cars ne pouvant s'arrêter se décourageront, les chambres d'hôtels se faisant de plus en plus rares, on aura une meilleure répartition du tourisme.

Il semble aujourd'hui aberrant de préconiser de telles mesures alors que de très nombreux transporteurs et de très nombreux hôteliers se plaignent du sous-emploi de leurs équipements. Mais ce sousemploi est dû au fait que, victime du mythe de l'expansion du tourisme, on a construit des hôtelsetdes équipementsdansle monde entier pensant que le tourisme allait se répartir géographiquement.

Or, cette répartition n'a pas été suffisante et ce sont les mégapoles qui ont surtout profité de l'expansion. Par ailleurs, on a trop construit sans tenir compte de la capacitéd'accueil des lieuxvisités. C'estcomme si on construisait un parking pour 5000 véhicules pour un théâtre de 500 places. II y a donc un rééquilibrage nécessaire à faire entre les équipements et le nombre de visiteurs que peut recevoir un lieu ou une ville sans être dégradé. Ce réequilibrage doit passer par la voie réglementaire qui permettra un bon aménagement. Il faudra donc une volonté politique. Il semble que certains pays aient choisi cette voie. Repensons au Cambodge et à Angkor Vat mentionné plus haut. Certains rares pays sont encore fermés au tourisme, c'est un extrême que nous n'approuvons pas, mais c'est donc possible.

Toutefois, la réponse sera surtout culturelle. Quandlesmoyens de transportétaient moins développés et qu'il était difficile de voyager, on pouvait en toute impunité publier les récits de voyages, vanter tel ou tel paysage et parler de sites exotiques enchanteurs.

A notre époque, quand on fait cela, on déclenche un énorme mouvement de foule. Tout le monde veut aller dans les mêmes endroits, où il fait bon vivre. Les transporteursorganisent des lignes. On y construit des aéroports et des hôtels, des tours operators commercialisent le sproduit $s$ et l'lle, la Côte ou la Province sont transformées de lieux de vies en produits touristiques, perdent leur åme et leur authenticité. Ceux qui les aimaient et les appréciaient les ont perdu à jamais; avec le nombre qu'entraîne le succès, le lieu disparaît.

Le désir d'aller dans les «sanctuaires*, provoqué par la publicité et la mode, est souvent malsain. Il procède du désir de dominer son voisin, de lui montrer que l'on fait ce qu'il n'a pas fait.

Peut-êtrefaudra-t-il montrer quel'homme qui a réussi n'est pas forcément celui qui fréquente un «resorts sous les tropiques, ou qui se trouve à Paris ou à Londres pour la «saison $\%$, mais celui qui reste volontiers dans son jardin avec sa famille ou qui se promène dans les rues de sa ville.

Ainsi, après avoir encouragé le mythe de l'exotisme que sous-tendait quelque peu l'idéologie colonialiste du siecle dernier, nous créerons le mythe de l'homme moderne qui reste au foyer. Ce sera lui le play boy dans le coup et non pas celui qui parcourt le monde en jet.

Alors, peut-être quand nous voyagerons, au lieu de pester contre l'inconfort des aéroports, des files d'attentes et des hôtels stéreotypés. Nous retrouverons les plaisirs qu'ont connus nos pères, grands-pères et autres ancêtres quand ils faisaient leur *tour comme on disait alors.

Il nous faudra peut-être *réapprendre» à voyager. Ne plus accepter d'être tassé dans un salon d'attente d'aerroport, puis dans un avion. Apprendre à renoncer plutôt que de mal profiter ou de mal utiliser tout ce que le monde met à notre disposition, comprendre que voyager n'est pas avaler des kilomètres et quela connaissance des autres ne se mesure pas au nombre des pays visités.

On est souvent déçu par les pays donton a rêvé et la sagesse voudrait sans doute qu'on est resté au niveau du rêve et que l'on ne voyage que quand c'est vraiment indispensable et non pas par désoeuvrement, par mode ou par incapacité à accepter le réel.

Mais, qui estprêtà renoncer à ses vacances?

Si on n'arrive pas à persuader le public de refuser d'être traité comme un troupeau dans les grandes destinations touristiques, un type de vision du monde sera irrémédiablement perdue. La vision est quelquechosed'éphémèremais qui perdure dans la conscience de celui qui a vu. Celui qui aura vu la Baie des Anges avec 50000 personnes, n'aura pas vu la même baie que celui qui l'a vu avec 500 personnes.

Il s'agit culturellement de faire comprendre que c'est avec 500 personnes que l'on perçoit tel paysage, et pas avec 5000 , et qu'il faut refuser d'être un des 5000 .

Cette réflexion n'a pas d'autre but que de signaler un danger qu'on n'ose pas souvent dénoncer. On met volontiers en avant les effets pervers du tourisme. Mais on parle très rarement des causes de ces effets pervers.

Il est possible que dans un proche avenir, nous soyons obligés d'agir pour limiter le succès et l'expansion du tourisme. $f$ 